\title{
Optimization algorithms in the design of switched-reluctance machines
}

\author{
Anastasia $V$. Shevkunova ${ }^{1, *}$ \\ ${ }^{1}$ Rostov State Transport University, 2,Rostovskogo Strelkovogo Polka Narodnogo Opolcheniya sq., \\ 344038, Rostov-on-Don, Russia
}

\begin{abstract}
Currently, the level of industrial development is determined by the intellectual capacity of the equipment used. To increase the energy efficiency of production in various industries, it is necessary to introduce automated electric drives based on promising switched-reluctance motors. The geometric parameters of the active part of the switched-reluctance motor were calculated. As a criterion, the maximum value of the average value of the electromagnetic moment for the switching period was taken as the basis. Optimal design was carried out by means of the computer-aided design program developed by the author in co-authorship. It, in turn, includes optimization algorithms based on stochastic and deterministic methods. The developed program for optimizing the active part of the switched-reluctance motor was implemented using the Matlab application software package, which directly interacts with the FEMM program for calculating and visualizing electromagnetic processes. Finding the most energy-efficient engine geometry was performed in two stages, in order to determine the geometric parameters of the engine tooth zone that have a dominant influence on the value of the electromagnetic moment, as well as to ensure a guaranteed finding of the global extremum of the target function.
\end{abstract}

\section{Introduction}

The Russian Federation has adopted a number of federal programs related to energy conservation, energy efficiency and the introduction of technologies necessary for the design and production of competitive high-tech products. The «Energy Strategy of Russia for the period up to 2035» states that the development of energy-saving and energy-efficient technologies in transport, housing and utilities, and industry is one of the key areas of Russia's development. As you know, more than $50 \%$ of the electricity generated in the Russian Federation is spent on powering electric drives of various technological installations. The transition from an unregulated electric drive to an adjustable one is one of the main ways to save energy, which, combined with automation capabilities, can ensure optimal use of electricity and other resources.

Adjustable electric drives are now constantly expanding their application areas. For a long time, the leading position in the field of adjustable electric drives was occupied by an asynchronous electric drive, thereby replacing the DC electric drive with an adjustable speed.

\footnotetext{
*Corresponding author: nastya3051990@mail.ru
} 
The reasons for the incident were the well-known shortcomings of the DC motor: the presence of a collector-brush unit that needed regular maintenance and, as a result, the low reliability of the machine, as well as the relatively high cost of the DC machine.

Due to the ever-increasing requirements for the technical and economic indicators of electric drives, the asynchronous electric drive does not always meet these high requirements (reliability, maintainability, cost, etc.), which leads to the search for an alternative replacement. Achievements in the field of power electronics and microprocessor technology allow us to consider as a competitor the switched-reluctance drive (SRD), which has received much attention in recent decades.

Automated drives based on switched-reluctance motors (SRM) have become an alternative to electric drives with AM for a number of applications both in mechanical engineering and in other areas of industry [1-4]. Recently, there have been quite a lot of published studies aimed at eliminating the existing shortcomings of electric machines of the switched-reluctance type and improving their energy efficiency [5-10].

For electric reciprocating drives of various industrial mechanisms, for example, in the oil industry, it is rational to use a cylindrical linear valve motor (CLVD) [11]. This type of electric machines is given a sufficient number of publications and they are gradually finding more and more use [12-14].

The structure of the SRD is quite simple, and this is one of its advantages. It consists of the motor itself-an inductor machine, an electronic switch connected to a rectifier in parallel with a capacitor (frequency converter) and controlled usually by a rotor position sensor via a control circuit.

Thanks to the following advantages of SRD, the scale and complexity of the task is formed. First, it is a fairly simple, technologically advanced and reliable engine design. Secondly, the electronic switch provides the supply of unipolar pulses to the motor phases, which makes it more reliable in comparison with a similar frequency converter for an asynchronous electric drive. Third, the weight and size and energy characteristics of the SRD are not inferior to and even surpass the frequency-controlled asynchronous electric drive. And, fourth, as an advantage, we can distinguish a wide variety of SRD structures, which makes it possible to use this type of drive for both low-speed and high-speed versions. From the above advantages, it follows that the SRD is a strong competitor of modern adjustable electric drives with traditional types of electric machines. However, despite a number of listed advantages, the widespread introduction of SRD has not yet occurred.

Specialists working in the field of electric drives explain this by the lack of necessary power electronics modules, controllers and the technological base for the production of these machines. The problem of optimal SRM design remains unsolved until the end. After all, it is the configuration of the active part of the machine that is responsible for the characteristics of the designed machine [15]. Thanks to the rapid development in the field of power electronics, computer technology, combined with optimization algorithms, it is possible to solve the main problems of this type of drive.

\section{Materials and research methods}

The purpose of the study is to improve energy efficiency, in particular, the operational performance of the system by increasing the average value of the electromagnetic moment. This goal was achieved by finding the optimal geometry of the gear zone of the engine in question. The solution was made by changing the design dimensions of the active part of the machine using optimization algorithms. The task can be defined as a single-criteria one from the variation of several geometric dimensions of the active part of the SRM. As the object under consideration, the engine with the following technical data was taken as the basis for the study (Table 1). 
Table 1. Technical data of the engine in question.

\begin{tabular}{|l|c|}
\hline \multicolumn{1}{|c|}{ Parameters } & Value \\
\hline Number of stator poles & 6 \\
\hline Number of rotor poles & 4 \\
\hline Number of phases & 3 \\
\hline Outer radius of the stator, mm & 74.5 \\
\hline Inner radius of the stator (along the grooves), $\boldsymbol{R 2 s}, \mathbf{m m}$ & $\mathbf{6 3 . 5}$ \\
\hline The width of the stator tooth crown, $\boldsymbol{b 1}, \mathbf{m m}$ & $\mathbf{1 8 . 2}$ \\
\hline The angular size of the stator tooth, $\boldsymbol{b 2 s}, \mathbf{d e g}$. & $\mathbf{0}$ \\
\hline Internal radius of the stator (along the crown of the teeth), mm & 42.2 \\
\hline Radius of the rotor (in half), mm & 33 \\
\hline The width of the rotor tooth crown, $\boldsymbol{b 1} \boldsymbol{r}, \mathbf{m m}$ & $\mathbf{1 7 . 3}$ \\
\hline Radius of the rotor (along the crown of the teeth), mm & 42 \\
\hline Air gap, $\boldsymbol{\delta}, \mathbf{m m}$ & $\mathbf{0 . 2}$ \\
\hline Shaft hole radius, mm & 17.5 \\
\hline The size of the magnetic core in the axial direction, mm & 140 \\
\hline Power, W & 4250 \\
\hline
\end{tabular}

Based on the theoretical and practical studies of this type of electric machine, in this study, as a material for the manufacture of the active part of the SRM, the electrotechnical sheet steel of the 2411 brand was chosen, and the coils located on the stator were taken from insulated copper wire.

As is known from the theory of electric machines, the main property of a magnetic core is a high magnetic permeability, which determines the ability of the material to support the propagation of a magnetic field, i.e., to create a magnetic flux with the lowest possible value of the magnetomotive force. Since the active part of the magnetic circuit is the most voluminous element of the structure, and it is subject to a large remagnetization due to the peculiarities of the engine operation. The latter circumstance causes additional losses on hysteresis and eddy currents, which can be quite large. Therefore, in order to reduce additional losses without increasing the brittleness of steel, the steel grade 2411 with a silicon content of $2.8-3.8 \%$ was selected for the study. This grade of steel is cold-rolled, which improves the magnetic properties of the material when it is magnetized along the rolling direction.

The magnetization curve of steel 2411 is shown in Figure 1.

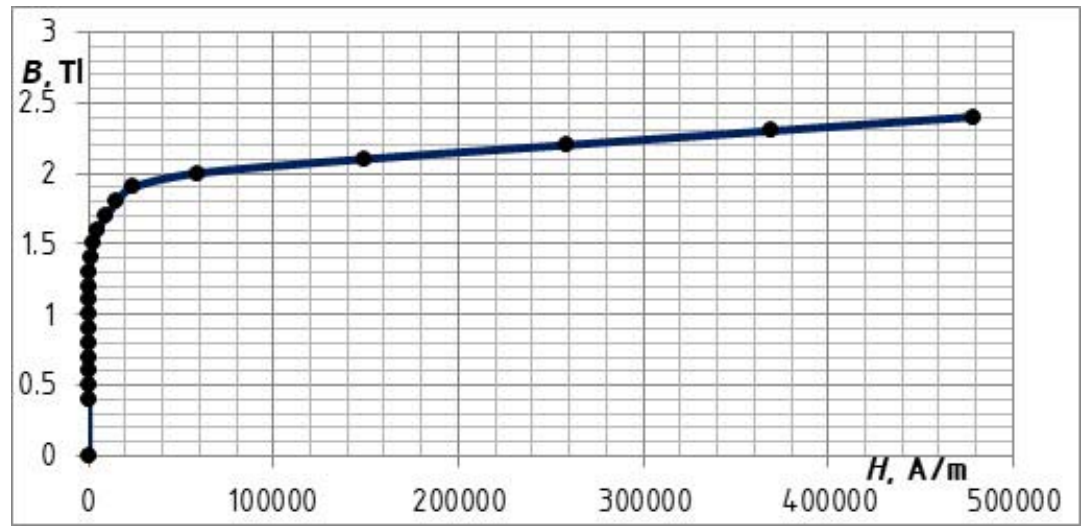

Fig. 1. Magnetization curve of steel 2411.

Calculations are made for the modes of starting and accelerating at low speeds of rotation of the SRM. For this mode, the torque on the rotor shaft is one of the most important indicators of the machine. The determination of the numerical value of the electromagnetic 
moment was carried out taking into account the saturation of the steel, since at the moments of start-up and overload, a significant saturation of the magnetic system is observed. Based on this, the solution of the selected problem was obtained by the finite element method using the FEMM 4.2 program. The following assumptions were made: the current in the active stator coil was kept constant in the so-called «current corridor» mode; phase switching was considered instantaneous; the supply network was assumed to be of infinite power.

The increase in the electromagnetic torque was obtained by changing the geometry of the active part of the engine using optimization algorithms based on the Nelder - Mead and Monte-Carlo methods (static tests). The geometric dimensions shown in bold in Table 1 were selected as the optimized parameters, and visual fragments of the geometry are shown in Figure 2.

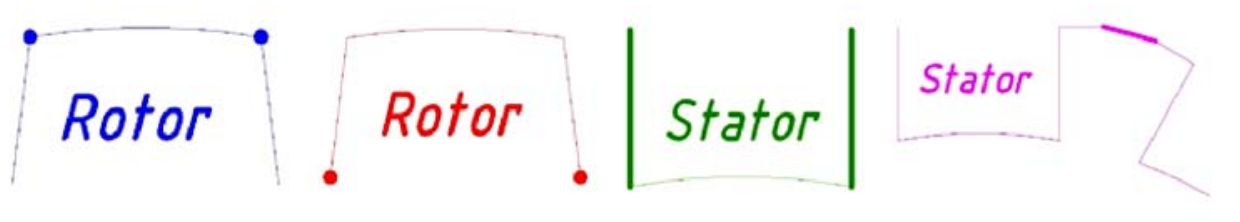

Fig. 2. Geometric fragments for optimization.

Optimization by the Monte-Carlo and Nelder - Mead methods was carried out in the following sequence.

The algorithm, based on the Monte-Carlo method, is based on the use of models of possible results. When creating these models, any parameter that has the nature of uncertainty is replaced by a range of certain values (the probability distribution).

By applying a probability distribution, variables can have different probabilities of consequences occurring. For example, this algorithm uses a normal distribution (or «Gaussian curve»), which has a symmetric shape in the form of a «bell», and there are other modifications of this method.

The main feature of the normal distribution is that all other laws tend to approach this distribution, i.e. the Gaussian law can be called the «limit law».

A continuous random variable $x$ has a normal distribution law (Gauss law) with parameters $a$ and $\sigma$ if its probability density is defined on the entire numerical axis.

According to the "Gaussian curve», the maximum is reached at the point $x=a$, i.e. at the middle of the distribution. This average value is called the mathematical expectation $(M)$. This means that the parameter a in the probability density of the Gaussian law is equal to the mathematical expectation of a random variable: $M(x)=a$.

There is also another necessary parameter-the standard deviation $(\sigma)$, which is defined as $\sigma^{2}=D(x)(D$ is the variance that characterizes the spread of a random variable relative to the average value). It is this value that characterizes the shape of the Gaussian curve. The lower the value of $\sigma$, the more accurate the result of the average value in the sample will be. If the standard deviations are large enough, then it is assumed that the average value lies in some range of numbers, and is not a specific number.

For this algorithm, the distribution of a random variable with an equal probability density was assumed. The principle of the algorithm is as follows.

One of the main optimization conditions is to set the number of parameters to be optimized (i.e., variables $-n)$ and set the constraints $\left(\mathrm{a}_{1 \min } \ldots \mathrm{a}_{\mathrm{nmin}} ; \mathrm{a}_{1 \max } \ldots \mathrm{a}_{\mathrm{nmax}}\right)$. After the initial conditions are formed to run the program, you need to set the number of calculations (NumCalc), which, as mentioned above, should be quite large. Next, a random number generator enters the process, which randomly throws the values of the parameters in the specified constraints according to the following formula: 


$$
a=a_{\min }+\left(a_{\max }-a_{\min }\right) \cdot \text { rand }
$$

where rand $-\mathrm{a}$ random number in the range from 0 to 1 .

At the next stage, the target function is calculated depending on the thrown parameter values: $C F_{t}=f\left(a_{t 1} \ldots a_{t n}\right)$. Thus, the actions will be repeated until the counter $t$ becomes greater than NumCalc: $t>$ NumCalc. The result of the optimization will be the values of a corresponding to the maximum (or minimum) of the objective function.

Optimization of the engine geometry was implemented using the developed program in the Matlab environment. The software package, combining the advantages of Matlab and FEMM, provides the possibility of accurate, high-quality, fast and automated modeling of the model. In our case, Matlab was used to calculate the coordinates of the characteristic points needed to create a geometric image of the SRM. Then, using the FEMM program, the torque on the motor shaft was calculated for each position of the rotor with a specified angular pitch. In the present case, the number of finite elements for the object under consideration was 1,876 . A fragment of the object under study with a grid of finite elements is shown in Figure 3.

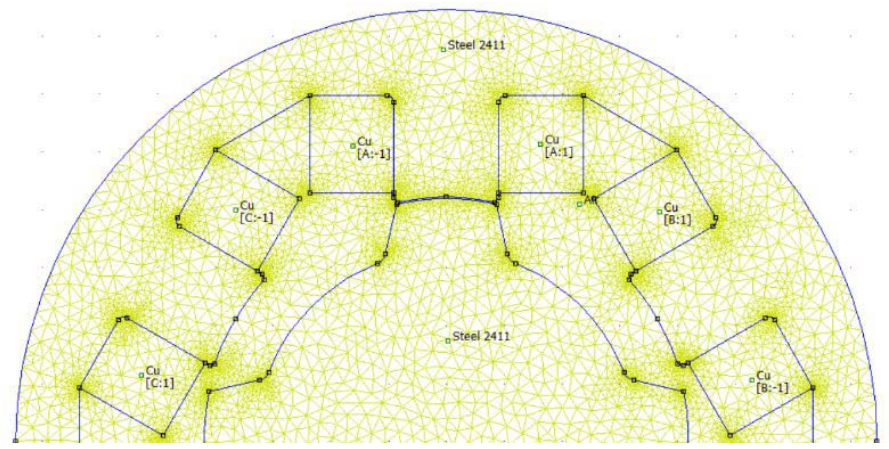

Fig. 3. SRM 6/4 in FEMM 4.2.

The calculation of the moment and forces by the FEMM program is based on the reduction of the Poisson formula to the problem of solving a system of algebraic equations, the number of which is proportional to the number of finite elements of the partition.

The magnetic field of the motor was assumed to be plane-parallel. In this case, only the component of the vector magnetic potential perpendicular to the plane in which the object field is considered is determined. After the parameters of the magnetic field are calculated the vector magnetic potential $\mathbf{A}$ and the vector of magnetic induction $\mathbf{B}$, using the ratio, the moments and forces acting in the SRM are calculated.

$$
\operatorname{rot} \mathbf{A}=\mathbf{B}
$$

where $\mathbf{B}$ - the magnetic induction vector.

Creating the optimal design of the active part of the engine under consideration, it is necessary to form the initial geometric dimensions and geometric size constraints, within which the search for the maximum of the average torque value will be carried out. To activate the program, you also need to specify the current value and the materials from which the design area of the motor is made. After setting the initial conditions for optimizing the geometry, the optimization method is selected, based on which the average torque on the motor shaft is calculated for each position of the rotor with a certain angular pitch. The moment was calculated using the derivative of the coenergy, which resulted in the dependence of the moment on the angle of rotation of the rotor $M=f(\Theta)$. To obtain the average value of the moment, this curve was numerically integrated by the trapezoid method. 
The calculation and optimization of the object under consideration were carried out in two stages. At the first stage, the moment calculations were performed with only two different variables, using the static test method (stochastic method). Further, at the second stage, the engine was optimized according to four parameters, at which the torque was maximum at the first stage. The last stage was the optimization of the object with narrower boundaries for calculation using the deformable polyhedron method, i.e. the obtained values were the result of the work carried out. The solution of the problem and the results obtained are presented in Table 2 .

Table 2. Problem solution and optimization result.

\begin{tabular}{|c|c|c|c|}
\hline $\begin{array}{c}\text { Variable } \\
\text { parameters }\end{array}$ & $\begin{array}{c}\text { Best combination of } \\
\text { design parameters. } \mathbf{~ m m}\end{array}$ & $\begin{array}{c}\text { Average value } \\
\text { moment. } \mathbf{~} \cdot \mathbf{m}\end{array}$ & $\begin{array}{c}\text { Increased torque } \\
\text { compared to the } \\
\text { riginal. \%o }\end{array}$ \\
\hline Initial data & \multicolumn{3}{|c|}{ The Monte-Carlo method } \\
\hline \multicolumn{4}{|c|}{12.591} \\
\hline$\delta ; b 2 s$ & $0.21 ; 3.37$ & 12.603 & 0.09 \\
\hline$\delta ; b 1 s$ & $0.23 ; 17.41$ & 12.612 & 0.17 \\
\hline$\delta ; b 1 r$ & $0.22 ; 18.9$ & 12.607 & 0.13 \\
\hline$\delta ; b 2 r$ & $0.2 ; 15$ & 12.623 & 0.25 \\
\hline$\delta ; R 2 s$ & $0.22 ; 65.61$ & 13.427 & 6.23 \\
\hline$\delta ; b 1 s ; b 2 r ;$ \\
$R 2 s$ & $0.22 ; 17.38 ; 11.26 ; 65.32$ & 13.64 & 7.69 \\
\hline \multicolumn{4}{|c|}{ The Nelder-Mead method (refinement) } \\
\hline $\begin{array}{c}\delta ; b 1 s ; b 2 r ; \\
R 2 s\end{array}$ & $0.2 ; 17.02 ; 11.27 ; 65.83$ & 13.87 & 9.22 \\
\hline
\end{tabular}

Figure 4 shows the torque curves of the engine for three cases: the initial one, after optimization by the Monte Carlo method for four variables, and the refined version after optimization by the Nelder-Mead method.

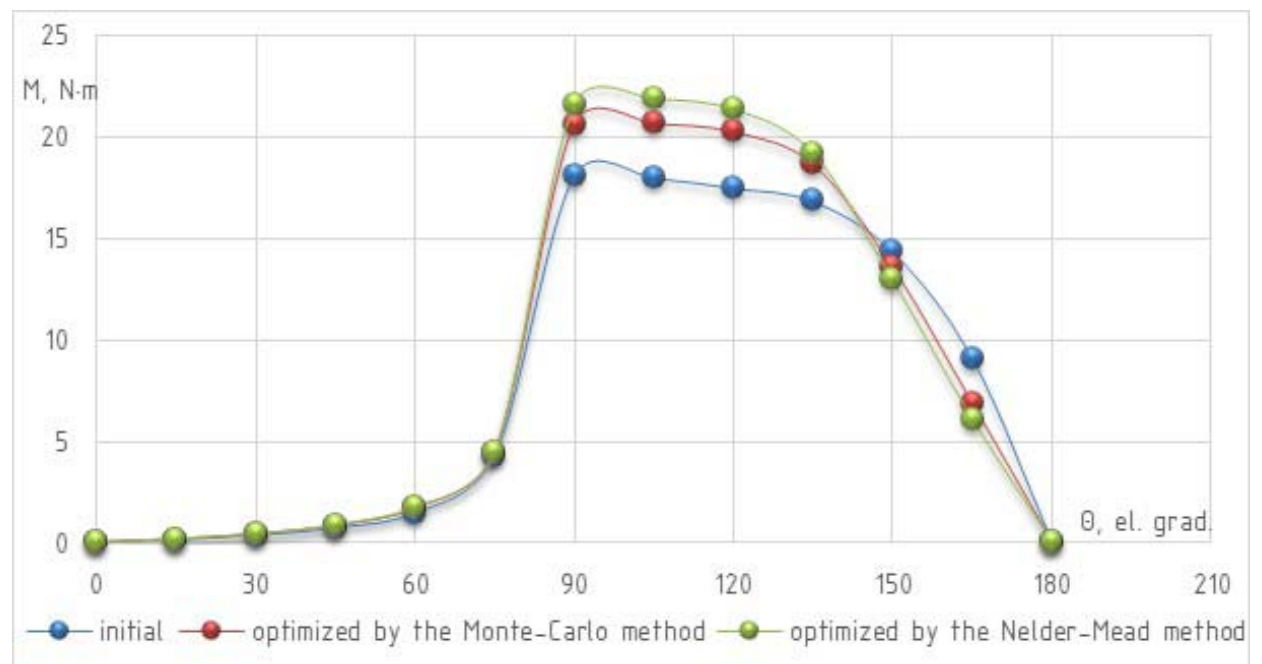

Fig. 4. Moment curves for three cases of the same phase.

Since the geometry of the original engine was changed after the optimization, there is a need for thermal calculation. The purpose of the work performed is to determine the temperature of the heating-limiting parts of the modified electric motor. 
Since the geometry of the original engine was changed after the optimization, there is a need for thermal calculation. The purpose of the work performed is to determine the temperature of the heating-limiting parts of the modified electric motor.

Using the FEMM program, the stationary thermal field of the source engine and the engine with the optimal geometry were calculated. To solve this problem, the following assumptions were made: the losses in the stator, rotor and windings were calculated based on the generally accepted method; the volume density of heat release in the motor windings was calculated from the effective current value; the thermal conductivity coefficient for all materials was assumed to be constant at any temperature.

Further, on the basis of the technical source data of the engine, its geometric dimensions, losses were determined, based on which the volume density of heat release of the stator, rotor and motor winding was calculated. As mentioned earlier, the magnetic system is made of 2411 steel, the winding is made of copper, the inter-turn insulation and the body insulation is glass-aluminum tape. In this study, the boundary conditions of the 3 rd kind-convection were chosen. The ambient temperature was assumed to be $20^{\circ} \mathrm{C}$. The resulting magnetic flux distribution is shown in Figure 5.

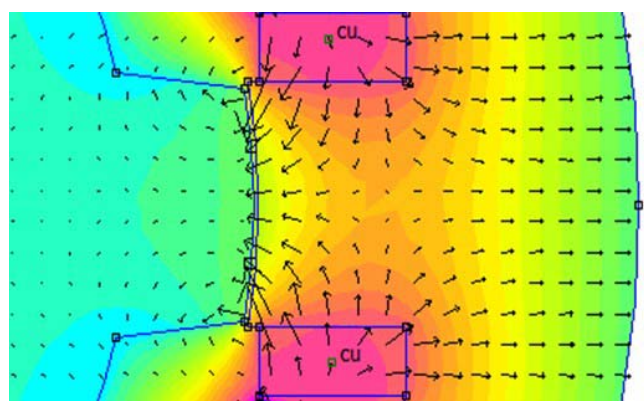

Fig. 5. Magnetic flux distribution.

\section{Consideration}

There are various recommendations and techniques for the design of valve-inductor machines, focused on various applications of the type. Some specifications and limitations may be specified in the design specification. In order to take them into account and at the same time ensure the best performance of the machine, it is advisable to use optimization algorithms when designing. Optimization methods and algorithms must meet the main criteria: the convergence of obtaining a solution with an iterative algorithm, the time to calculate the final result, and achieving the required accuracy. As shown by the study, in order to most accurately solve the optimization problem, it is necessary to synthesize deterministic algorithms (for example, the Nelder - Mead method) with random search methods (for example, the Monte-Carlo method).

\section{Conclusion}

In the course of the study, a single-criteria optimization was carried out, where the target function was the average value of the electromagnetic moment, and the geometric dimensions of the active part of the engine were taken as variables. The result of the optimal design was an increase in the average electromagnetic torque by $9.22 \%$. At the same time, the overall dimensions, i.e. the external diameter of the core and the length of the magnetic core in the axial direction remained unchanged. The thermal calculation of the machine was also carried 
out, which showed that the excess temperature of the resulting model does not exceed $2.5 \%$, which is considered correct and acceptable.

\section{References}

1. L. Proskuryakova, S. Filippov, Energy technology Foresight 2030 in Russia: an outlook for safer and more efficient energy future 75, 2798-2806 (2015) doi: 10.1016/j.egypro.2015.07.550

2. M. Hamoudaab, A. Menaemc, H. Rezkde, M. Ibrahimfgh, L. Számel, An improved indirect instantaneous torque control strategy of switched reluctance motor drives for light electric vehicles 6, 709-715 (2020) https://doi.org/10.1016/j.egyr.2020.11.142

3. Q. Yang, R. Wang, M. Ma, S. Yang, X. Zhang, A fault tolerant switched reluctance motor drive for electric vehicles under multi-switches open-fault conditions 114, 113761 (2020), https://doi.org/10.1016/j.microrel.2020.113761

4. F. Daldaban, N. Ustkoyuncu, A novel linear switched reluctance motor for railway

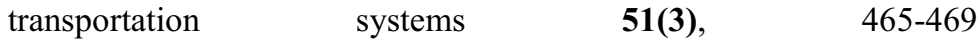
https://doi.org/10.1016/j.enconman.2009.10.009

5. A. Petrushin, E. Miroshnichenko, M. Tchavychalov, Journal of Engineering and Applied Sciences 10(5), 102-106 (2015)

6. A. Petrushin, M. Tchavychalov, E. Miroshnichenko, International Journal of Power Electronics and Drive System (IJPEDS) 6(1), 86-91 (2015)

7. N. Grebennikov, 26th International Workshop on Electric Drives: Improvement in Efficiency of Electric Drives 8743038 (2019)

8. N. Grebennikov, T. Talakhadze, A. Kashuba, 26th International Workshop on Electric Drives: Improvement in Efficiency of Electric Drives, IWED 2019 - Proceedings 8664226 (2019)

9. N. Grebennikov, A. Lebedev, Journal of Engineering and Applied Sciences 13(21), 8935-8938 (2018)

10. A. Petrushin, A. Kashuba, Russian Engineering Research 38(9), 705-706 (2018)

11. S. Shutemov, E. Chabanov, A. Shevkunova, A. Shapshal, T. Talakhadze, Reduction of the pull effect of a cylindrical linear synchronous motor 157, 01015 (2020) https://doi.org/10.1051/e3sconf/202015701015

12. U. Thakar, V. Joshi, U. Mehta, V.A. Vyawahare, Fractional-Order PI Controller for Permanent Magnet Synchronous Motor: A Design-Based Comparative Study, 553-578 (2018) https://doi.org/10.1016/B978-0-12-816152-4.00018-2

13. C. Xiang, F. Liu, H. Liu, L. Han, X. Zhang, Nonlinear dynamic behaviors of permanent magnet synchronous motors in electric vehicles caused by unbalanced magnetic pull 371, 277-294 (2016) https://doi.org/10.1016/j.jsv.2016.02.015

14. Guan Xiao-Cun, Lei Bin, Li Zhi-Yuan, Zhao Ran, Research on Performance High-speed Multi-stage Cylinder Linear Induction Motor 16, 1904-1912 (2012) doi:10.1016/j.egypro.2012.01.291

15. A. Petrushin, A. Shevkunova, Investigation study of geometric dimensions of the magnetic system of the switched-reluctance machine influence on magnetic moment 313(1), 012009 (2018) https://doi.org/10.1088/1757-899X/313/1/012009 